\title{
Current Scenario of Dentistry and Role of Dental Institutions in Providing Education and Care in India
}

${ }^{1}$ Vikas Gupta, ${ }^{2}$ DR Prithviraj, ${ }^{3}$ Ninad Muley

\begin{abstract}
Introduction: India is the largest democracy and the second most populated country in the world. Because of this, Indian healthcare industry is experiencing quick transformation owing to the increasing demand for quality healthcare. This huge market of dental industry in India has also made a remarkable impact on the dental education.
\end{abstract}

Search strategy: PubMed (using medical subject headings) database was searched in the English language by using the following combinations of keywords: dental education, dental colleges, dentist: population ratio, dental council of India. Other articles were identified from the reference lists of the articles. The data from the identified studies were collected and reviewed.

Discussion: Through this article admission procedure, fees structure, dental curriculum, work culture and pay scale of dental institutes has been discussed. Existing dental taskforce and dentist population ratio of India has been discussed. Measures to be taken to improve the scenario of oral healthcare for the Indian population have been enlightened.

Keywords: Dental colleges, Dentist: population ratio, Dental council of India, Rural dental health.

How to cite this article: Gupta V, Prithviraj DR, Muley N. Current Scenario of Dentistry and Role of Dental Institutions in Providing Education and Care in India. J Postgrad Med Edu Res 2015;49(3):111-114.

\section{Source of support: Nil}

Conflict of interest: None

\section{INTRODUCTION}

Though India is poised to become the leader in the global economy in this century, its population of more that 1.22 billion, will challenge both the medical and

\footnotetext{
${ }^{1}$ Senior Resident, ${ }^{2}$ Professor and Head, ${ }^{3}$ Senior Lecturer

${ }^{1}$ Department of Oral Health Science Centre, Postgraduate Institute of Medical Education and Research, Chandigarh, India

${ }^{2}$ Department of Prosthodontics, Government Dental College and Research Institute, Bengaluru, Karnataka, India

${ }^{3}$ Department of Prosthodontics, Sri Sai College of Dental Surgery, Hyderabad, Telangana, India
}

Corresponding Author: Vikas Gupta, Senior Resident Department of Oral Health Science Centre, Postgraduate Institute of Medical Education and Research, Chandigarh, India Phone: 82883807178, e-mail: dr_vikas.gupta@yahoo.com dental healthcare systems. Thus, the current scenario of dental education and practice in India has some serious limitations which need to be sorted out to meet the oral healthcare needs of the population. Diseases, such as dental caries, periodontal problems, poor oral hygiene and oral cancers have always been major public health issues in India. It is well known that poor socioeconomic status (SES) provides negative impact on favorable dental health awareness, attitude and oral hygiene behavior.

Dentistry in some form has been practiced since the era of the Indus valley civilization. Ancient medical literatures, such as the Ayurveda and Susrutasamhita described treatments of diseases of the oral cavity and emphasized the importance of tongue hygiene. ${ }^{1}$ Dental education in India was established 91 years ago, when the first dental college was started in Calcutta by Dr Ahmed in 1920. This institution offered a diploma of licentiate in dental science upon successful completion of a 2 years program. Incidentally, this was a private college which was handed over to the West Bengal government in the year 1949. In this era, dental education was provided in government colleges only and there were no private colleges. The first private dental college was established in the 1960 s. $^{2}$ Currently, the growth of private dental colleges has far exceeded the number of governmentaided colleges. The aim of this literature review is to provide an overview about the role of dental institutions in providing the education and care in India.

\section{Search Strategy}

PubMed (using medical subject headings) database was searched in the English language by using the following combinations of keywords: dental education, dental colleges, dentist: population ratio, dental council of India. Other articles were identified from the reference lists of the articles, as well as from early online articles. The data from the identified studies were collected and reviewed.

\section{DISCUSSION}

The level of dental education and current scenario of dentistry in India has been discussed. Through this article admission procedure, fees structure, dental curriculum, work culture and pay scale of dental institutes has been discussed. Existing dental taskforce and dentist 
population ratio of India has been discussed. Measures to be taken to improve the scenario of oral healthcare for the Indian population have been enlightened.

\section{The Dental Council of India, Dental College Systems, Admission Procedures and Fee Structure}

Efforts of various organizations, like World Health Organization (WHO), Indian Dental Association (IDA) and Dental Council of India (DCI), to increase awareness of oral hygiene have provided major contribution in establishing today's developing dental industry. The DCI was established as a result of the Dentist Act of 1948, which was intended to regulate dental practice and promote scientific advances. Dental Council of India is still the premier governing body of dental education in India. Its responsibilities include the regulation of dental education, profession, and ethics and liaising with the government to obtain administrative approval for dental college and higher educational courses. Dental colleges in India fall into one of three major categories and their admission procedure also varies accordingly: ${ }^{3}$

Government dental college as a part of a government university: The admission is done strictly on the basis of merit. The students have to qualify either a state level examination or a national level entrance exami-nation. Both these examinations are government-administered entrance examinations. The fee which is charged is very nominal and there is also provision for scholarships for the students.

Private dental colleges affiliated with a government university: in these colleges, admission is possible through either a government administered state level examination or an examination conducted by a private organization for all the colleges under a single government university. There are also some seats reserved for the management quota. The fee is less for candidates qualifying through the government administered exam whereas the students taking the management seats have to pay more fees.

Private colleges under a private university: In these colleges, the admission is through either examination conducted by the university itself or through the management quota. In some colleges, seats may be reserved for NRI candidates also. Fee structure of these colleges is also higher as compared to that of government colleges.

\section{Dental Curriculum, Work Culture and Pay Scales (Faculty)}

Currently, the duration of undergraduate dental training (bachelor of dental surgery, BDS) in India is 5 years, including 1 year of compulsory internship. The students rotate through various dental specialties after the completion of the formal course work and examinations given during the first half of the 4 years of the program. During the first 2 years of the program, students enroll in a core curriculum that includes human anatomy, human physiology, biochemistry, pathology, microbiology, pharmacology and dental anatomy. In this same period, students have to attend some preclinical classes by working on the dummies with artificial teeth or on extracted teeth or on ideal molds and models. These exercises not only improve their confidence to work in clinical condition but also make them more familiar with various dental materials. In the 3rd and 4th years, apart from classes corresponding to courses in the core curriculum that include general medicine, general surgery, and all the dental specialties, students also complete rotations in various clinical departments. They will typically spend a fixed period of time at each rotation (usually 1 month) to improve their clinical skills before moving on to other departments.

The duration of postgraduate course (master of dental surgery, MDS) is 3 years in India. The programs foster development of critical analysis, research skills, and advanced clinical skills that will be recognized for specialist registration. Dental Council of India is the regulatory body for dental courses (BDS and MDS) and various institutes have to adhere to the regulations provided. Master of dental surgery is available in specialities of:

- Oral medicine and radiology

- Conservative dentistry and endodontics

- Public health dentistry

- Periodontology

- Oral and maxillofacial surgery

- Prosthodontics and crown and bridge

- Pedodontics and preventive dentistry

- Orthodontics and dentofacial orthopedics

- Oral pathology and microbiology

The government colleges have been in existence for a number of years and have always provided treatment at very nominal charges. Thus, they help to cater to the oral healthcare needs of the poor section of the population and have always had an abundance of patients. Most of the private colleges also provide dental treatment at very subsidized rates and some even provide free treatment, thus making oral healthcare available to the poor population. Due to the initial paucity of funds allocated by the government for the dental sector, the government colleges were restricted as far as procuring the best materials and equipments were concerned. Because of an increasing competition between the government and private sector, the government colleges are also forced 
to improve their equipments and infrastructure and now many of the government colleges have been well equipped.

The government institutions offer a standardized pay scale for its entire faculty based on the position occupied by the faculty: that of a lecturer, reader, professor of a department. The salary is, thus, decided by the pay commission. The private colleges do not have a standardized pay scale and is highly dependant on the demand and supply ratio. This is a direct effect of the commercialization of the profession caused by the mushrooming of private colleges. Thus, there is need to establish an organization which would remove disparity in the salary scales even in private colleges, and thus avoid variation in the salary scales.

\section{The Existing Dental Taskforce and Dentist Population Ratio}

There is a staggering of 294 dental colleges in India, 253 are private colleges. Out of the total 30,000 graduates passing out of the dental colleges every year (Dental Council of India's figure-29,530/Year), only 2,000 pass out from government institutions. More than 2,800 postgraduate students are enrolled every year in various specialties of dentistry (DentalCouncil of India's figure-2,681/Year). ${ }^{4}$ When we compare the dentist: population ratio of India to that of America, the population of America is nearly 33 crores, and there were 108 dental colleges. However, considering the dentists: population ratio and to cut the number of fresh dentists graduating every year, the number of dental colleges in America are now reduced to 58 , producing approximately 2,240 dentists. ${ }^{5}$ Thus, comparing to the ratio of dentists: population of America, India could have only 119 colleges producing approximately 12,000 dentists, against the existing 294 colleges. World Health Organization recommends, dentist to population ratio of 1:7500. Dentists-to-population ratio of India, which was 1:300,000 in the 1960's, stands at 1:10,000 today. ${ }^{6}$ Moreover, the distribution pattern of the dental colleges across various states of India is uneven (Table 1). This situation leads to unequal distribution of dentists across the states affecting their dentist: population ratio. ${ }^{7}$

Nowadays, dental colleges are providing treatment not only in the college premises but also at the community level to benefit a larger section of the population. Camps are regularly conducted in the rural areas to provide oral healthcare for the rural population. In these camps, apart from dental examination, basic dental treatment is also provided. If there is a need for any complex procedures, the patients are referred to the college for the same. Many of the colleges are also equipped with mobile dental vans,
Table 1: Distribution pattern of dental colleges across various states

\begin{tabular}{llll}
\hline State & Colleges & State & Colleges \\
\hline Karnataka & 43 & Himachal Pradesh & 5 \\
Maharashtra & 28 & Bihar & 7 \\
Uttar Pradesh & 28 & Chhattisgarh & 5 \\
Andhra Pradesh & 20 & West Bengal & 3 \\
Tamil Nadu & 17 & Puducherry & 2 \\
Haryana & 11 & Delhi & 3 \\
Rajasthan & 11 & Goa & 1 \\
Punjab & 12 & Odisha & 4 \\
Madhya Pradesh & 11 & Assam & 1 \\
Kerala & 17 & Uttarakhand & 2 \\
Gujarat & 9 & Jammu and Kashmir & 2 \\
\hline
\end{tabular}

thereby providing effective treatment to the population. Mobile dental vans are an effort whereby dental treatment is provided at the patient's doorstep. Thus, the doctor goes to the patient and provides the treatment, which might become the need of the hour in the future.

\section{Thus, the question arises: Are we really short of dentists in India?}

To answer this query, one should understand the distribution of dentists in India. The problem lies at the level of distribution rather than the number of dental surgeons. About $80 \%$ of dentists work in major cities in India; compared to the population where more than $70 \%$ of the Indians reside in the rural areas. Very little oral healthcare services are provided in the rural areas, however; oral healthcare seeking behavior is also very low, especially among the rural population. A mismatch exists between oral health professionals and the population they serve. ${ }^{8}$ Even with a workforce of $1,18,000$ qualified dentists in the country, the most basic oral health education and simple interventions are also not available to vast majority of rural population and that is the misery of the whole scenario.

Thus, reach and quality of public health services have been below desirable standards. Eighty to $85 \%$ of people are spending money from their pocket. Majority of the Indian population belong to the lower socioeconomic strata and reside in the rural sector. Thus, the oral health seeking behavior is very low in India. Although the huge unmet treatment needs exist; striking inequality in delivery system and absence of an adequate communityoriented prevention system is further aggravating the lack of reach of dental facilities in rural parts of India. In the last financial year, only $4.1 \%$ of the total GDP was allocated to health-related expenditures in India, whereas other smaller Southeast Asian countries with smaller population allocate nearly the same amount or more for health related activities. ${ }^{9}$ Thus, a lot needs to be done by 
the government to improve the access to oral healthcare for the general population and also take measures to change its policies toward the oral health sector.

\section{Measures to be taken to Improve the Scenario of Oral Healthcare for the Indian Population}

World Health Organization focused its attention on oral health in 1994 and chose the theme 'Oral Health for Healthy life' for World Health Day. ${ }^{10}$ Since 1994, the WHO has emphasized on the lack of oral healthcare facilities in the rural areas especially in developing countries and the need to improve the provision of oral healthcare to the rural sector.

Thus to equalize the uneven distribution of dentists between the urban and rural areas, the government has to take adequate measures to promote the dentists wishing to practice in the rural areas. This can be done by attracting the graduates from both private and government colleges to cater to the rural population by providing jobs at every level of the rural healthcare integrated network. Further, the government can provide incentives to those establishing practice in rural areas by providing space for the set-up of dental clinics at a subsidized rate. Further, to provide efficient healthcare at the PHC level, availability of dental materials and equipments should be improved. However, frequency of visits to a dental clinic is unexpectedly low in the rural area due to poor health education. This problem also needs to be rectified by various means of education program. The government as well as private companies should make an effort to start insurance policies to cover for the dental healthcare needs of the population, which would benefit both the rural and the urban population. Such insurance policies are already functional in many western countries like the USA, and cover many of the basic dental procedures. An effort should also be made to start a National Health Scheme to cover dental treatment. National health service (NHS) is functional in many countries, such as England under which the people are provided dental health through their health insurance policies. It is the publicly funded healthcare system. Primarily funded through the general taxation system, the system provides healthcare to every legal resident in the country, with most services free at the point of use. ${ }^{11}$

Thus, to conclude to improve the dentist: population ratio in rural areas, the government has to take adequate measures to promote the dentists wishing to practice in the rural areas. Further, the adequate utilization of graduates from both the government and private sector would be required for the equalization of the dentist to population between the rural and urban sector and for the betterment of the dental profession as a whole.

\section{REFERENCES}

1. Hoffmann-Axthelm W. History of dentistry. Chicago: Quintessence Publishing Co; 1981;36-46.

2. Tandon $S$. Challenges to the oral health workforce in India. J Dent Educ 2004;68:28-33.

3. BDS. course regulations. New Delhi: Dental Council of India, June 1983.

4. Database of Dental Council of India. Cited on 2014 June 1. Available at: http://www.dciindia.org/search.aspx.

5. McKinnon M, Luke G, Bresch J, Moss M, Richard W. Valachovic. Emerging Allied Dental Workforce Models: Considerations for Academic Dental Institutions. J Dent Educ 2007;71:11.

6. National Oral Health Policy: Prepared by core committee, appointed by the Ministry of Health and Family Welfare, 1995.

7. Jain H, Agarwal A. Current Scenario and Crisis Facing Dental College Graduates in India. J Clinical and Diagnostic Res 2012;6:1-4.

8. Sivapathasundharam B. Dental education in India. Indian J Dent Res 2007;18:93.

9. Database of world health organization. Cited on 2014 June 1. Available at: http:/ /www.who.int/countries/ind/en/

10. Oral Health: ICMR Bulletin, Volume 24, April, 1994 ICMR, New Delhi.

11. Database of Wikipedia with title National Health Service (England) Available at: http://en.wikipedia.org/wiki/ National_Health_Service_28\% England 29\%. 